\section{Prevalência de fluorose dentária em escolares de 12 anos de idade, Ouro Preto/MG - 2003}

\section{Prevalence of dental fluorosis in 12 year-old schoolchildren, Ouro Preto/MG - 2003}

\section{Silvana Fioravante Braga Barros ${ }^{1}$ \\ Divane Leite Matos ${ }^{2,3}$}

${ }^{1}$ Prefeitura Municipal de Ouro Preto/ Curso de Especialização em Saúde Coletiva da Pontifícia Universidade Católica de Minas Gerais

${ }^{2}$ Núcleo de Estudos em Saúde Pública e Envelhecimento (NESPE). Centro de Pesquisas René Rachou da Fundação Oswaldo Cruz/ Faculdade de Medicina da Universidade Federal de Minas Gerais

${ }^{3}$ Secretaria Estadual de Saúde de Minas Gerais/Coordenadoria de Saúde Bucal

\section{Resumo}

Objetivo: verificar a prevalência da fluorose dentária em escolares de 12 anos de idade em Ouro Preto. Metodologia: estudo seccional incluindo 248 crianças selecionadas aleatoriamente em 11 escolas do município. A amostra foi calculada de acordo com a metodologia descrita no Projeto SB2000. Os exames foram realizados por cirurgião-dentista treinado e calibrado. Os índices utilizados para medir a fluorose foram: índice de Dean e índice comunitário de fluorose. Resultados: De 248 crianças selecionadas, $159(64,1 \%)$ participaram deste estudo. A prevalência de fluorose (índice de Dean) encontrada foi de $11,4 \%$, sendo composta pela condição muito leve $(10,1 \%)$ e leve $(1,3 \%)$. A condição moderada e severa de fluorose não foi encontrada. Os dentes mais acometidos por fluorose foram os pré-molares, molares, incisivos e caninos. Não houve diferença entre os sexos quanto ao grau de fluorose, e o índice comunitário de fluorose foi de 0,29 . Conclusão: a fluorose em crianças de 12 anos não é um problema de saúde pública em Ouro Preto, dado que a prevalência não é alta e quase todos os casos encontrados são muito leves.

Palavras-chave: Fluorose dentária. Escolares. Saúde bucal. Epidemiologia.

Correspondência: Silvana Fioravante Braga Barros. Rua Cristina 1192 apto. 1401, Bairro Santo Antônio, Belo Horizonte, Minas Gerais, CEP:30330-130.E-mail: slbarros@globo.com 


\section{Abstract}

Objective: to find out the prevalence of dental fluorosis in 12-year-old schoolchildren in Ouro Preto. Methodology: cross-sectional study including 248 children randomly selected from 11 schools in the town. The sample was calculated according to the methodology described in Project SB2000. Exams were undertaken by a trained and calibrated dentist. Dental fluorosis was evaluated by the Dean index and index of community fluorosis. Results: 159 (64.1\%) children, among the 248 who were selected, participated in the study. The prevalence of fluorosis (Dean index) found was $11.4 \%$, and it was found to be a very mild $(10.1 \%)$ or mild (1.3\%) condition. Moderate and severe fluorosis were not found. The teeth most affected by fluorosis were pre-molars, molars, incisors and canines. There was no difference between genders as to the degree of fluorosis, and the index of community fluorosis was 0.29 . Conclusion: fluorosis in 12 year-old children is not a public dental health problem in Ouro Preto, as the prevalence is not high and nearly all cases are very mild.

Keywords: Dental fluorosis. Schoolchildren. Oral health. Epidemiology.

\section{Introdução}

Fluorose dentária é uma opacidade do esmalte provocada pela ingestão de quantidades excessivas de flúor (exposição crônica) durante o período de formação dos dentes. As manifestações vão depender da quantidade de flúor ingerida, do tempo de exposição, da idade, do peso e do estado nutricional do indivíduo ${ }^{1}$.

Este defeito no esmalte ocorre muitas vezes devido à concentração de flúor na água de consumo, quando ingerida, principalmente em crianças de até 5 anos de idade. A dentição permanente é mais acometida do que a decídua, devido ao período de formação do dente decíduo ser mais curto e a placenta ser uma barreira para a transferência de altas doses de fluoretos para o bebế2

Segundo Buzalaf et al. ${ }^{3}$ e Moysés et al. ${ }^{4}$, a prevalência de fluorose aumentou, principalmente as formas leve e muito leve, tendo sido observada durante os últimos 30 anos tanto em comunidades com água fluoretada como em comunidades que consomem água não fluoretada. Sendo necessário um monitoramento rigoroso dos teores de flúor na água de abastecimento, e que medidas para controlar a fluorose devem levar em conta a autopercepção do problema pela população $0^{4}$.

Cangussu et al. ${ }^{5}$, fizeram uma revisão crítica sobre fluorose dentária no Brasil e concluíram que ela constitui um relevante problema para a saúde bucal coletiva e que seriam necessários estudos epidemiológicos para acompanhar a tendência da prevalência e a severidade da doença. Constataram que as formas brandas são comuns onde há água de abastecimento público fluoretada; que é necessário a vigilância das proporções de flúor na água de consumo; que a quantidade máxima permitida em águas minerais, bebidas enlatadas, refrigerantes, sucos e chás devem ser regulamentadas e apresentadas em rótulos. Os cremes dentais devem ter os seus teores de flúor verificados periodicamente, bem como difundir a necessidade do uso de pequena quantidade de creme dental no ato de 
higienizar os dentes e a importância da supervisão dos pais no momento da escovação em crianças menores de 7 anos.

Além da cárie dentária, a fluorose também passou a ser uma condição que merece estudos para avaliar sua tendência, já que se tem observado uma exposição crescente ao flúor. Devido não somente à sua presença na água de abastecimento público, mas também nas pastas dentais, nos bochechos e nas aplicações tópicas, atividades instituídas nos programas de prevenção, além dos suplementos vitamínicos com flúor.

O presente trabalho tem como objetivo verificar a prevalência de fluorose dentária em escolares de 12 anos em Ouro Preto.

\section{Material e Métodos}

\section{Área estudada}

Ouro Preto é uma importante cidade histórica de Minas Gerais e situa-se a 100 km de Belo Horizonte, capital do Estado. Possui uma população total de 66.277 habitantes, sendo que 56.292 (84,9\%) residem na área urbana do município onde este trabalho foi desenvolvido ${ }^{6}$. A atividade econômica de Ouro Preto gira em torno do turismo e de indústrias metalúrgicas como a Alcan (Alumínio do Brasil) e a CVRD (Companhia Vale do Rio Doce), entre outras?

A Alcan é uma indústria de alumínio e de embalagem e foi em Ouro Preto a primeira metalúrgica a ter o processo completo, da extração da bauxita até a produção do alumínio primário. Em 1979, foi implantado um programa de controle ambiental, onde foram instalados equipamentos e adotadas medidas antipoluentes, reduzindo suas emissões em mais de $80 \%$, entre esses poluentes o flúor ${ }^{8}$. A água de abastecimento em Ouro Preto não é fluoretada.

\section{População estudada}

Participaram deste trabalho crianças de 12 anos de idade matriculadas no $1^{\circ}$ semestre escolar de 2003, em escolas da rede pública e privada da sede do município de Ouro Preto/Minas Gerais. A amostra foi calculada seguindo a metodologia descrita no Projeto SB2000 do Ministério da Saúde ${ }^{9}$. O tamanho da amostra ajustado para populações finitas segundo a idade/grupo etário e o tipo de município por macro-região foi de 248 escolares. Maiores detalhes estão descritos no Manual do Coordenador do Projeto $\mathrm{SB} 2000^{10}$. A amostra foi realizada em dois estágios: seleção das escolas e seleção das crianças participantes do estudo.

No primeiro estágio, o critério de inclusão utilizado foi: "escolas de ensino fundamental” freqüentadas por crianças de 12 anos de idade, situadas na sede do município. E o critério de exclusão foi: "escolas de ensino fundamental”, apenas com séries de $1^{\underline{a}}$ a $4^{\underline{a}}$, e escolas especializadas em crianças portadoras de algum tipo de deficiência. Das 27 instituições de ensino localizadas na sede do município, onze (09 da rede pública e 02 da rede particular) preencheram o critério de inclusão.

No segundo estágio, as crianças foram selecionadas a partir de uma lista única, fornecida pela Secretaria Municipal de Educação de Ouro Preto, de todos os escolares de 12 anos matriculados nas onze escolas (que preencheram o critério de inclusão). Para esta seleção foi utilizada a técnica da amostra aleatória simples. $\mathrm{Na}$ listagem, cada escolar foi numerado e os números escolhidos foram gerados aleatoriamente através do programa Epi-Info versão $6.04 \mathrm{a}^{11}$. O exame clínico das crianças foi consentido pelos pais ou responsáveis através da assinatura do Termo de Consentimento Livre e Esclarecido. Aquelas cujos pais ou responsáveis não consentiram, foram excluídas da amostra e não foram substituídas.

Este estudo foi submetido e aprovado pelo Comitê de Ética em Pesquisa (CEP) da Pontifícia Universidade Católica de Minas Gerais (PUC/MG). A realização desta pesquisa também foi autorizada (por escrito) pela Secretaria Municipal de Saúde de Ouro Preto. 


\section{Coleta de dados}

Os dados foram coletados através de exames clínicos realizados por um único examinador (cirurgião-dentista) e um dos autores deste trabalho, que foi treinado e calibrado para verificar a concordância intraexaminador ${ }^{12}$. No processo de calibração foi feita a avaliação da concordância dos resultados, por meio da aplicação da estatística Kappa $^{13}$, sendo a concordância considerada muito boa (Kappa $=0,91$ intervalo de confiança a $95 \%=0,56-1,25$ ).

Durante a coleta de dados foram realizados exames em duplicata a partir de cada décima criança examinada, para avaliação da concordância dos diagnósticos durante a realização dos exames. Os resultados indicaram que a concordância do examinador foi também boa (Kappa $=0,89$ intervalo de confiança a $95 \%=0,49-1,15$ ) durante a coleta de dados.

Os exames foram realizados nas próprias escolas, sob luz natural, utilizando-se gaze esterilizada, abaixadores de língua e equipamento de proteção individual. Todos os dentes permanentes presentes foram examinados. De acordo com o Manual do Examinador - Projeto SB2000 ${ }^{14}$, para a avaliação da condição individual foram considerados apenas os dois dentes mais afetados (se esses dois dentes mais afetados não estiverem comprometidos de modo semelhante, o valor do menos afetado entre os dois foi registrado). Os dados foram anotados em ficha clínica elaborada para este estudo.

Para avaliação da fluorose dentária foram utilizados o índice de Dean ${ }^{15}$ e o índice comunitário de fluorose - ICF ${ }^{16}$.

\section{Análise dos dados}

A análise dos dados foi descritiva, baseada em proporções e freqüência absoluta e relativa simples para descrever o índice de fluorose (índice de Dean) e os dentes mais acometidos.

A condição questionável de fluorose não foi incluída no cálculo do índice de Dean; apenas as condições muito leve, leve, moderada e severa foram consideradas, pois os dentes classificados na condição questionável seriam aqueles duvidosos quanto à existência ou não de fluorose dentária, casos que não se enquadram totalmente na classificação de normal e que apresentam algumas alterações no esmalte dentário, mas que também não justificaria a classificação leve ${ }^{15}$.

O processamento dos dados e a análise estatística foram realizados no programa Epi - Info versão $6.04 \mathrm{a}^{11}$.

\section{Resultados}

De 248 escolares selecionados, 159 $(64,1 \%)$ foram submetidos ao exame. A perda amostral foi de $35,9 \%(n=89)$, representando aquelas crianças em que os pais ou responsáveis não consentiram ou que estavam ausentes no momento do exame. Do total de participantes deste estudo ( $\mathrm{n}=$ 159), $49 \%$ eram do sexo masculino e $51 \%$ do sexo feminino. Destas, $96,2 \%(n=153)$ estudavam em escolas públicas e 3,8\% (n = 6) em escolas privadas da sede do município de Ouro Preto.

Quatro crianças foram classificadas como condição do dente igual a excluído (sem possibilidade de exame) dentro dos parâmetros propostos pelo índice de Dean, representando $2,4 \%$. Na Tabela 1, estão apresentados os valores relativos à prevalência de fluorose na amostra examinada, segundo os critérios de Dean.

Do total de crianças examinadas, a maioria $(52,2 \%)$ não apresentou fluorose (os dentes foram classificados na condição normal) e $34 \%$ foram classificadas na condição questionável. Esta condição se enquadra nos casos duvidosos quanto à existência ou não de fluorose dentária. O índice de prevalência de fluorose (índice de Dean) foi de $11,4 \%$, sendo composto pela condição muito leve $(10,1 \%)$ e leve $(1,3 \%)$. As condições moderada e severa não foram encontradas. Em relação ao índice comunitário de fluorose foi achado um valor igual a 0,29.

Os grupos de dentes mais afetados foram os pré-molares $(47,3 \%)$, primeiros 
Tabela 1 - Distribuição da prevalência de fluorose dentária, segundo os critérios de Dean em crianças de 12 anos. Ouro Preto, 2003. Table 1 - Distribution of the prevalence of fluorosis according to the Dean index in 12 year-old children. Ouro Preto, 2003.

\begin{tabular}{lcc}
\hline Condição & $\mathrm{n}$ & $\%$ \\
\hline Sem fluorose & 137 & 86,2 \\
Normal & 83 & 52,2 \\
Questionável & 54 & 34,0 \\
Com fluorose & $\mathbf{1 8}$ & $\mathbf{1 1 , 4}$ \\
(índice de Dean) & & \\
Muito leve & 16 & 10,1 \\
Leve & 2 & 1,3 \\
Moderada & - & - \\
Severa & - & - \\
Excluída & 4 & 2,4 \\
Total & 159 & 100,0 \\
\hline
\end{tabular}

molares $(19,7 \%)$, incisivos $(17,1 \%)$, segundos molares $(9,4 \%)$ e caninos $(6,5 \%)$.

\section{Discussão}

O índice de fluorose encontrado nas crianças de 12 anos de idade da cidade de Ouro Preto foi de $11,4 \%$, enquanto que em cidades paulistas sem flúor na água, este índice foi de 4,9\% em 1998 e 0,6\% em $2002^{17,18}$.

Apesar do município de Ouro Preto não possuir água fluoretada, a prevalência de fluorose encontrada foi semelhante àquela achada em alguns municípios com água fluoretada. No levantamento epidemiológico realizado no estado de São Paulo em $1998^{17}$, a prevalência nos municípios que possuíam água fluoretada foi igual a $14,9 \%$, sendo composta pela condição muito leve $(11,7 \%)$, leve $(2,3 \%)$, moderada $(0,8 \%)$ e severa $(0,1 \%)$ e, no outro estudo também realizado em São Paulo ${ }^{18}$ em 2002, nos municípios com flúor na água de abastecimento este índice foi igual a $13,8 \%$, sendo a condição muito leve $(10,9 \%)$, leve $(2,2 \%)$, moderada $(0,6 \%)$ e severa $(0,1 \%)$. Prevalência semelhante a que encontramos em nosso estudo $(11,4 \%)$ foi também verificada em crianças de 12 anos de Bauru/SP (11,9\%), sendo que $6,9 \%$ apresentavam fluorose mui- to leve, $4,0 \%$ leve e $1,0 \%$ moderada ${ }^{19}$. Em estudo realizado em Belo Horizonte (cidade que possui flúor na água de abastecimento desde 1975), no ano de 1995, a prevalência de fluorose foi igual a $12,7 \%^{20}$.

Em relação aos dentes mais afetados por fluorose dentária, os nossos resultados foram semelhantes ao de dois outros estudos ${ }^{1,16}$ realizados na população brasileira.

O índice comunitário de fluorose apresentou valor igual a 0,29 . Este resultado foi semelhante ao de Silva \& Paiva $^{20}(0,28)$ e Alcântara $^{21}(0,25)$, em regiões que possuíam flúor na água de abastecimento.

O presente trabalho possui algumas limitações que merecem ser discutidas. As duas principais referem-se à perda amostral e ao possível viés de classificação do examinador. A perda amostral atuou no sentido de aumentar o número de crianças do sexo feminino e estudantes de escolas públicas. Entre os 248 escolares selecionados, a proporção do sexo masculino era de $52,8 \%$ e a do feminino de $47,2 \%$, e as crianças que efetivamente participaram deste trabalho foram iguais a 49 e $51 \%$, respectivamente. Quanto ao tipo de escola, a relação de escolares selecionados foi de $93,1 \%$ em escolas públicas e de 6,9\% em particulares, e entre as crianças que participaram foi igual a 96,2 e $3,8 \%$, respectivamente. Outro fato a ser considerado, é o alto percentual de crianças classificadas na condição questionável, comparado a outros estudos ${ }^{17-21}$, o que pode sugerir um viés na classificação do examinador.

Embora a fluorose dentária não pareça ser um problema de saúde pública em Ouro Preto, a prevalência encontrada mostrouse semelhante à de alguns municípios com flúor na água de abastecimento público, tanto em relação ao índice de Dean como ao índice comunitário de fluorose $\mathrm{e}^{17-21}$. É importante observar a qualidade da água consumida e os diversos meios de acesso ao flúor, bem como avaliar a possibilidade de ocorrência de fluorose causada por poluição industrial. A partir daí implantar um programa adequado de fluoretação e vigilância dos teores de flúor no ar e na água, 
com o objetivo de monitorar as concentrações e obter do método, o maior benefício em termos de prevenção de cárie com o menor risco possível de fluorose.

A fluorose dentária não pode ser atribuída unicamente a fluoretação da água de abastecimento público, porém é necessário pesquisar o efeito interativo das diferentes fontes de flúor, como a ingestão de parte da quantidade de dentifrícios usados nas escovações, o "efeito halo" (produtos produzidos em regiões fluoretadas e consumidas em regiões não fluoretadas), o consumo de água engarrafada, leite em pó, medicamentos, dentre outros ${ }^{22}$.

\section{Conclusão}

A fluorose dentária em crianças de 12 anos não é um problema de saúde pública em Ouro Preto, dado que a prevalência não é alta e quase todos os casos encontrados são muito leves. Apesar deste achado, fato importante é que este município não possui água de abastecimento público fluoretada. Desta forma, torna-se necessária a implantação de sistemas de vigilância em saúde para monitorar o acesso que esta população tem às diversas formas de fluoreto, assim como as tendências na prevalência de fluorose.

\section{Referências}

1. Frazão P, Peverari AC, Forni TIB, Mota AG, Costa LR. Fluorose dentária: comparação de dois estudos de prevalência. Cad Saúde Pública 2004; 20(4): 1050-8.

2. Horowitz HS. Indexes for measuring dental fluorosis. Pub Hlth Dent 1986; 46: 179-83.

3. Buzalaf MAR, Cury JC, Whitford GM. Fluoride exposures and dental fluorosis: a literature review. Rev FOB 2001; 9(2): $1-10$.

4. Moysés SJ, Moysés ST, Allegretti ACV, Argenta M, Werneck R. Fluorose dental: ficção epidemiológica? Rev Panam Salud Publica/Pan Am J Public Health 2002; 12(5): 339-46.

5. Cangussu MCT, Narvai PC, Fernandez RC, Djehizian V. A fluorose dentária no Brasil: uma revisão crítica. Cad Saúde Pública 2002; 18(1): 7-15.

6. Fundação João Pinheiro - FJP. Disponível em http:// www.fjp.gov.br

7. Universidade Federal de Ouro Preto. Disponível em http://www.ufop.br.

8. Alcan. Disponível em http://www.alcan.com.br.

9. Brasil. Ministério da Saúde. Área Técnica de Saúde Bucal. Projeto SB2000 - Condições de Saúde Bucal da População Brasileira no Ano de 2000. Brasília; 2000.

10. Brasil. Ministério da Saúde. Projeto SB2000. Condições de Saúde Bucal da População Brasileira no Ano de 2000. Manual do Coordenador. Brasília; 2001a.

11. Dean AG, Dean JA, Coulombier D, Brendel KA, Smith DC, Burton AH, et al. Epi Info, version 6.0: a Word Processing Database and Statistics Program for Epidemiology on Microcomputers. Atlanta, Georgia: Centers for Disease Control and Prevention; 1994.
12. Brasil. Ministério da Saúde. Projeto SB2000. Condições de Saúde Bucal da População Brasileira no Ano de 2000. Manual de Calibração de Examinadores. Brasília; 2001b.

13. World Health Organization. Calibration of examiners for oral health epidemiological surveys. Geneva: ORH/EIS/ EPID; 1993.

14. Brasil. Ministério da Saúde. Projeto SB2000. Condições de Saúde Bucal da População Brasileira no Ano de 2000. Manual do Examinador. Brasília; 2001c.

15. Organização Mundial da Saúde. Levantamento Epidemiológico Básico de Saúde Bucal: Manual de Instruções. Genebra 4a ed. 1997.

16. Fejerskov O, Manji F, Baelum V, Möeler IJ. Fluorose dentária: um manual para profissionais de saúde. São Paulo: Santos; 1994.

17. São Paulo. Secretaria Estadual de Saúde. Faculdade de Saúde Pública. Universidade de São Paulo. Levantamento epidemiológico em saúde bucal: Estado de São Paulo, 1998: Relatório. São Paulo: SES-SP e FSP - USP; 1999.

18. São Paulo. Secretaria Estadual de Saúde. Centro Técnico de Saúde Bucal. Universidade de São Paulo. Faculdade de Saúde Pública. Núcleo de Estudos e Pesquisas de Sistemas de Saúde. Condições de Saúde Bucal no Estado de São Paulo em 2002: Relatório Final. São Paulo: SES-SP e FSP-USP; 2002.

19. Buzalaf MAR, Bastos JRM, Lauris JRP, Almeida BS, Aquilante AG. Association between the early use of toothpaste and other variables with dental fluorosis: a transversal retrospective study. Rev Fac Odontol Bauru 2002; 10(3): 196-200. 
20. Silva ALCC, Paiva SM. Ocorrência de fluorose dentária em escolares de Belo Horizonte. Revista do CROMG 1995; I(2): 49-53.

21. Alcântara CM. Prevalência de fluorose dental em escolares de Curitiba. Rev ABO Nacional 1998; 6(5): 304-7.
22. Peres SHCS, Bastos JRM. Perfil epidemiológico de cárie dentária em crianças de 12 anos de idade, residentes em cidades fluoretadas e não fluoretadas, na região CentroOeste do Estado de São Paulo, Brasil. Cad Saúde Pública 2002; 18(5): 1281-8.

Recebido em: 10/03/05

Versão reformulada reapresentada em: 28/09/05

Aprovado em: 28/09/05 